\title{
The physical mechanisms that initiate and drive solar eruptions
}

\author{
Guillaume Aulanier \\ Observatoire de Paris, LESIA, CNRS, UPMC, Univ. Paris Diderot, \\ 5 place Jules Janssen, 92190 Meudon, France \\ email: guillaume.aulanier@obspm.fr
}

\begin{abstract}
Solar eruptions are due to a sudden destabilization of force-free coronal magnetic fields. But the detailed mechanisms which can bring the corona towards an eruptive stage, then trigger and drive the eruption, and finally make it explosive, are not fully understood. A large variety of storage-and-release models have been developed and opposed to each other since 40 years. For example, photospheric flux emergence vs. flux cancellation, localized coronal reconnection vs. large-scale ideal instabilities and loss of equilibria, tether-cutting vs. breakout reconnection, and so on. The competition between all these approaches has led to a tremendous drive in developing and testing all these concepts, by coupling state-of-the-art models and observations. Thanks to these developments, it now becomes possible to compare all these models with one another, and to revisit their interpretation in light of their common and their different behaviors. This approach leads me to argue that no more than two distinct physical mechanisms can actually initiate and drive prominence eruptions: the magnetic breakout and the torus instability. In this view, all other processes (including flux emergence, flux cancellation, flare reconnection and long-range couplings) should be considered as various ways that lead to, or that strengthen, one of the aforementioned driving mechanisms.
\end{abstract}

Keywords. Solar corona, prominences, coronal mass ejections, MHD

\section{Introduction}

Eruptive prominences are large clouds of magnetized plasma, which are ejected from the low solar corona into interplanetary space, in the form of Coronal Mass Ejections (CMEs). They can erupt either from within active regions, or from long filament channels. During the eruption, the system accelerates up to typical velocities of $100-1000 \mathrm{~km} / \mathrm{s}$ (although slower and faster CMEs also exist) while flare loops always form in the wake of the eruption (even though they can be hard to see in weak events).

Since the low corona is a sufficiently collisional plasma, its evolution can be studied in the frame of MHD. Also, the ratio between thermal and magnetic pressure is there very small, i.e. $\beta \ll 1$. Therefore, the magnetic energy dominates all other forms of energy in the source regions of solar eruptions (see Forbes 2000, Table 2). Currentfree (potential) magnetic fields correspond to the minimum magnetic energy for a given distribution of magnetic flux through the dense photosphere. Since the photospheric flux distribution does not significantly change during the time-scales of eruptions, and since the powering of eruptions requires the magnetic energy to decrease, the coronal magnetic field must therefore be highly non-potential prior to eruption onset, i.e. it must contain strong electric currents. Due to the slow evolution of the photospheric magnetic field (as compared to typical coronal velocities), currents which are injected into the corona must accumulate slowly, such that the coronal field evolves quasi-statically, as a sequence of force-free equilibria. The triggering of CMEs therefore requires the coronal field to reach some threshold above which the balance between magnetic pressure (which points 
upward) and magnetic tension (which points downward) is broken. When the system suddenly enters a regime in which the pressure dominates, it can erupt in a catastrophic way, leading to a CME. The resulting ideal expansion of the magnetic field, as well as the resistively driven magnetic reconnection in the current layer that forms in the wake of the expanding system, both contribute to decrease the magnetic energy. These arguments are the root of the "storage-and-release" MHD models for solar eruptions.

Even though it is now widely accepted that solar eruptions are due to such a violent destabilization of previously energized coronal magnetic fields, the detailed mechanisms which bring a system into an eruptive stage, and which eventually drive the eruption, are not yet fully understood. A large variety of storage-and-release models has been put forward in the past decades (see Forbes et al. 2006; Schmieder et al. 2013, for two extensive reviews that also describe observations). Firstly, most of these models nicely describe many observed aspects of solar eruptions. Therefore it is difficult to estimate their respective merits solely based on observational criteria. Secondly, the models qualitatively share many common physical ingredients. So they may be difficult to distinguish from one another. So, to date, two questions remain open: Which physical mechanisms drive prominence eruptions? Which solar drivers can gradually bring stable prominences to eruptive states?

This paper aims at reviewing the existing storage-and-release models that are realistic enough, in terms of the solar physical conditions, and at considering them all together in a common frame, so as to bring some up-to-date answers to the two aforementioned questions. So this review focuses only on the onset and driving mechanisms of eruptions, not on their ensuing development in the large-scale corona and in the heliosphere.

\section{Non-equilibrium and instability electric-wire models}

The oldest prominence eruption model that remains considered to date is the loss-ofequilibrium model, that was initially put forward in the physical paradigm of electricwires, and that was further proven to occur in fully 3D MHD simulations.

\subsection{Straight-wire geometry}

The original model was developed in 2D, in cartesian geometry (van Tend \& Kuperus 1978; van Tend 1979; Molodenskii \& Filippov 1987; Filippov \& Den 2001). The set-up consists of a line current $I$ that is inserted at some height $z=h$ above the photospheric plane, $z=0$, an ambient coronal field $B_{\mathrm{ex}}$, and a so-called "image current" $-I$ is added at $z=-h$ to emulate one effect of photospheric line tying, i.e. so that the photospheric magnetic field does not change when $h$ changes. The resulting coronal magnetic field consists of a detached plasmoid (or flux rope) that mimics a prominence that is embedded in a coronal arcade, and whose apex is located at $z=h$.

In the "electric paradigm", the equilibrium of the system results from the competition between two Laplace forces, namely the downward force that $B_{\text {ex }}$ excerts on the coronal line current, and the upward force generated by the repulsion of the two line currents. In the "MHD paradigm", the former corresponds to restraining magnetic tension of the potential field overlying the flux rope, and the latter to magnetic pressure that results from the increase of the magnetic field strength below the coronal line current induced by the photospheric boundary.

With these settings, the equilibrium curve $h(I)$ has a critical point $\left(I_{c} ; h_{c}\right)$, beyond which the line-current $I \geqslant I_{c}$ cannot stay in equilibrium and must move to infinite $z$. The altitude $z=h_{c}$ of this critical point is given by the height at which $B_{\text {ex }}(z)$ starts to drop faster than $z^{-1}$. 
The cartesian model has been refined several times, e.g. by giving a finite width to the coronal current, by taking into account the conservation of magnetic flux during the eruption, and by treatig the line-tying at the photospheric part of the flux rope. The latter yields the formation of a vertical current sheet below the flux rope during its eruption (Martens \& Kuin 1989; Amari \& Aly 1990). This current sheet exerts an extra restraining force on the line current, such that the flux rope cannot move to infinity, but finds a new equilibrium position at finite $z$ (Forbes \& Isenberg 1991). In 2D, a full eruption requires the dissipation of this current sheet by sufficiently fast magnetic reconnection (Lin \& Forbes 2000). But this may not be required in 3D. This is in line with analytical MHD considerations on the energy of fully open (so unreconnected) magnetic fields. Indeed this energy is infinite in 2D cartesian geometry, while it remains finite in $2 \mathrm{D}$ axisymmetric spherical systems and in all 3D geometries (Aly 1984, 1991; Sturrock 1991).

\subsection{Curved-wire geometry}

The model has also been investigated in 2.5D axisymmetric (toroidal) geometry. In a first approach, the coronal line-current is replaced by a detached ring-current at some height above the photospheric spherical surface (Lin et al. 1998). If an image current is added below the photosphere so that the coronal arcades surrounding the flux rope are line-tied, the same repulsive and restraining forces as discussed above contribute to the force balance. However, a new repulsive force (which the current exerts on itself due to its bending) comes into play. This curvature (or "hoop") force is radially outward directed and can be balanced by an external magnetic field, $B_{\text {ex }}$ (Shafranov 1966; Chen 1989; Titov \& Démoulin 1999).

In these spherical models, the requirement for magnetic reconnection below the rope as identified in cartesian geometry (Lin \& Forbes 2000) still holds, but it is less important because the rope can rise ideally to tens of solar radii before the Laplace force of the vertical current sheet can halt the eruption.

In a second approach, half of the ring-current of radius $R$ is emerged above a planar photosphere, and the other half located below the photosphere somehow plays the role of the image current. With these settings, the untied ring-current can freely expand radially, as a result of a so-called "torus instability". This instability occurs when the restoring force due to the external field drops faster with the altitude than the hoop force. For external poloidal fields (i.e. perpendicular to the current) with $B_{\text {ex }} \sim R^{-n}$, the instability threshold is given by $n_{c} \sim 3 / 2$ (Bateman 1978; Kliem \& Török 2006).

Qualitatively similar instability thresholds have been identified when the line-tying of the ring-current is treated, through the addition of multiple image current segments in the model (Isenberg \& Forbes 2007; Olmedo et al. 2013b).

\subsection{Discussion on electric-wire models}

The cartesian and the axisymmetric models had initially been developed separately. The former studied the conditions for "loss-of-equilibria", and the latter calculated onset criteria for "instabilities". Both approaches were recently revisited by Démoulin \& Aulanier (2010). Non-circular current paths were later considered (Olmedo \& Zhang 2010; Olmedo et al. 2013a,b). All geometries were shown to share almost the same analytical equations, and therefore the same physics. It was then proposed to join both approaches in a single "torus instability" mechanism.

This model has been criticized by several MHD physicists. Indeed the physical simplifcations of the electric-wire paradigm, and the qualitative nature of their link with the (correct) MHD paradigm, are a priori quite disputable. Nevertheless, the analytical elecric-wire predictions for eruptive thesholds have been found to match the onset 
of eruptions in some line-tied MHD simulations. Those include suspended flux ropes in 2.5D (Forbes 1990) and in 3D (Inoue \& Kusano 2006; Nishida et al. 2013), and fully 3D line-tied flux ropes (Roussev et al. 2003; Török \& Kliem 2005, 2007; Schrijver et al. 2008; Török et al. 2010; Jiang et al. 2013). So the electric-wire model was found to be consistent with its correct MHD treatment. But even then, some questions were left open. Indeed, even if all the aforementioned simulations correctly prescribed force-free flux ropes as initial conditions, firstly all but one used analytical flux rope solutions from Titov \& Démoulin (1999) that contain very idealized current distributions (much simpler than those produced by solar MHD processes [see e.g. Aulanier et al. 2005], which are themselves more compatible with photospheric observations [see e.g. Schmieder \& Aulanier 2012; Georgoulis et al. 2012]), and secondly these ropes were already unstable, so that their pre-eruptive evolution was not self-consistently treated.

In spite of all these issues, the torus instability was found to occur in some recent MHD simulations in which 3D flux ropes were gradually formed by photospheric drivers that mimic solar processes (see Aulanier et al. 2010; Fan 2010, as described further below). So the torus instability appears as a robust process to initiate and drive solar eruptions.

\section{MHD models based on increasing manetic pressure}

Any realistic eruption model must involve non-potential pre-eruptive coronal fields. There are various ways to generate them, as listed below. Some models investigated the role of the increasing magnetic pressure alone to drive an eruption.

\subsection{Axial flux increase}

The first models that were developed in the correct MHD paradigm were analytical and two-dimensional. There the prominence axis was oriented perpendicularly to the $2 \mathrm{D}$ plane of the models, and the magnetic shear was substituted for the electric current $I$ as a primary variable. But in the absence of a self-consistent way to prescribe increasing magnetic shear along the prominence, these models rather prescribed the prominence axial magnetic field (or flux) as a free parameter. The stability properties of the modeled systems were analyzed, in pretty much the same way as in the electric-wire models. Equilibrium curves were identified, and the lack of existing solutions were found for specific parameters, in particular for strong axial fields and when thermal pressure was taken into account (Low 1977; Birn et al. 1978; Heyvaerts et al. 1982; Zwingmann 1987).

These models remained theoretical, until the development of the flux-insertion method through magneto-frictional numerical relaxations in 3D (van Ballegooijen 2004). This novel approach allowed to model observed prominences and to find some eruptions, by "manually" inserting axial fluxes of different prescribed magnitudes ( $\mathrm{Su}$ et al. 2011).

The early 2D models and the recent $3 \mathrm{D}$ ones qualitatively interpreted their modeled eruptions as evidences for losses of equilibria that could occur when the ratio $\mathfrak{R}$ of the axial prominence flux to the overlying arcade flux exceeds some unidentified threshold (as discussed by Heyvaerts et al. 1982; Green et al. 2011).

It is only very recently that Kliem et al. (2013) performed new detailed analysis of the $3 \mathrm{D}$ models. They found that the eruption onset condition matches the threshold as predicted by the electric-wire models, namely the torus instability. This result is important in two ways. Fistly it shows that, even though the ratio $\mathfrak{R}$ is defined from the right MHD paradigm, its unclear condition for eruptiveness has to be substituted by the clearer criterion for torus instability, even if that one comes from the disputable electric-wire paradigm. Secondly, this result provides one more case of torus instability in numerical simulations. 


\subsection{Line-tied shearing and twisting motions}

The development of 3D line-tied MHD simulations showed that, when the system is driven by horizontal photosphetic motions, the axial flux cannot increase arbitrarily. Two situations were identified.

Firstly, if shearing or twisting flux tubes are restrained by strong non-moving overlying arcades in 3D, the axial flux eventually saturates. Then the system can either remain stable (Antiochos et al. 1994; DeVore \& Antiochos 2000; Aulanier et al. 2002) or eventually develop a kink instability that subsequently disrupts the whole configuration (Amari \& Luciani 1999). Secondly, if the overlying arcades are either too weak or also sheared or twisted, the whole system starts to expand. This bulging increases the length of the field lines, which in turn reduces the electric currents that have been induced by the photospheric motions. Analytical arguments (Aly 1985; Klimchuk \& Sturrock 1989; Sturrock et al. 1995) and numerical simulations (Mikic \& Linker 1994; Roumeliotis et al. 1994; Amari et al. 1996a,b; Aulanier et al. 2005) have shown that, in ideal MHD, the expansion-driven current decrease eventually dominates the shear-driven current increase. This effect prevents the magnetic field from reaching any loss of equilibrium.

In all 3D cases, no undriven expansion and therefore no eruption occurs. There are two counter-examples only, in 3D (Török \& Kliem 2003; Rachmeler et al. 2009). But those may not be applicable to prominence eruptions. Indeed the related loss of equilibrium there develops when the flux rope has strongly expanded, long before the eruption. In $2 \mathrm{D}$, shearing motions can easily produce eruptions if reconnection is allowed (see Mikic \& Linker 1994; Amari et al. 1996a; Jacobs et al. 2006, that are further discussed below). But this behavior has never been reproduced in 3D, except maybe in Archontis \& Hood (2008). All these results suggest that, in general, in 3D, simple line-tied shearing/twisting motions alone are not sufficient to drive an eruption. Nevertheless, line-tied motions provide a natural process to enhance the departure from non-potentiality that is required to power prominence eruptions.

\subsection{Twisted flux emergence}

Electric current and magnetic pressure can also be directly injected into the corona by the emergence through the photosphere of twisted flux ropes that rose through the convection zone (Emonet \& Moreno-Insertis 1998; Jouve \& Brun 2009) .

Some "kinematic flux emergence" simulations do achieve this. There the emergence is prescribed as time-dependent boundary conditions for the magnetic field in a linetied photospheric boundary, and the whole flux rope can be allowed to emerge from the photospheric boundary into the corona. Such simulations indeed lead to eruptions (Fan \& Gibson 2004; Amari et al. 2004, 2005; Fan \& Gibson 2007; Fan 2010). A clear result came from the careful analysis of some of those. There, eruptions have been unambigously shown to be attributed to the torus instability, as shown by Fan \& Gibson (2007) and later by Fan (2010). The former and latter constitute the first and third report, respectively, of a simulation that involved a torus-unstable flux rope that was gradually formed in the corona, and not prescribed as initial conditions as in the first MHD simulations of the torus instability.

Unfortunately, simulations of twisted flux emergence through a stratisfied medium (hence, non-kinematic emergence) show that, due to the weight of photospheric plasma which is trapped in its lower windings the flux rope hardly emerges as a whole (Fan 2001; Magara \& Longcope 2001; Archontis et al. 2004, 2009) as it does in the kinematic models. Unless the flux rope is not strongly curved (e.g. as in MacTaggart \& Hood 2009c), the only way for the lower part of the flux rope to emerge is to dispose of the dense plasma trapped in the photospheric dipped portions of the field. According to the "resistive flux 
emergence model" this may take place through magnetic reconnection photospheric Uloops (e.g. Pariat et al. 2004; Isobe et al. 2007). This difficulty still raises questions about the results of the kinematic simulations.

Nevertheless, a few non-kinematic simulations of flux emergence have successfully produced eruptions, using different codes and initial conditions (Manchester et al. 2004; Archontis \& Hood 2008; Archontis \& Török 2008; MacTaggart \& Hood 2009b; Archontis \& Hood 2012). But the physical mechanism that drive eruptions in these simulations remains unclear. Some self-induced shear flows in the photosphere may cause eruptions (Manchester et al. 2004). Magnetic reconnection with an ambient horizontal coronal field seems to trigger eruptions (Archontis \& Török 2008; MacTaggart \& Hood 2009b), like in the breakout model (see Sect. 4.2). But eruptions are not always successful with this process (MacTaggart \& Hood 2009a; Leake et al. 2010). The development of low-altitude magnetic reconnection within the emerging fields could also cause, or at least contribute to, the eruption of a newly-formed flux rope (Manchester et al. 2004; Archontis \& Hood 2008), like in the tether-cutting model (see Sect. 4.1). Finally, the relative strength of the overlying confining arcades as compared to that the emerging rope appears determining (Archontis \& Hood 2012), maybe like in the axial-flux increase models (see Sect. 3.1). So more investigation is required in terms of physical analysis. One other issue concerns the too small sizes of the modeled flux ropes in these simulations, relative to the thickness of the modeled photosphere.

\section{MHD models based on decreasing magnetic tension}

Instead of increasing the current to a value $I \geqslant I_{c}$ an alternative approach is to reduce the restraining tension of coronal arcades which overlie initially stable current-carrying magnetic fields. Most eruption models actually fall into this class.

\subsection{Tether-cutting}

Magnetic tension can decrease due to the breakdown of ideal MHD in the vertical currentsheet that forms within a shearing arcade (Amari \& Aly 1990; Forbes \& Isenberg 1991), resulting in magnetic reconnection that eventually forms flare loops and ribbons in the wake of the CME, i.e. below the current-carrying field lines. This non-ideal effect creates and feeds a twisted envelope around the initial current-carrying fields, from the flux of the overlying arcades. So the flare reconnection is an efficient process for reducing the downward tension of the arcades: it can "cut the tethers" (Sturrock 1989). This process is self-sustaining, since the more the flux rope rises during the eruption, and the more reconnection happens, the weaker is the restraining tension, so the more the flux rope can rise (Moore \& Roumeliotis 1992; Moore et al. 2001; Nishida et al. 2013). Thus, in principle, it can become explosive.

The tether-cutting effect alone has indeed been shown to trigger and to drive eruptions in 2.5D cartesian (Amari et al. 1996a) and axisymmetric (Mikic \& Linker 1994; Jacobs et al. 2006) MHD simulations. Early tether-cutting reconnection has also been found to sustain the formation of twisted envelopes in 3D MHD simulations of sheared arcades (DeVore \& Antiochos 2000), of flux cancellation (Aulanier et al. 2010) and of kinematic flux emergence (Fan 2010). But it did not cause the eruption, when there was one, in any of these simulations. Also this reconnection there tends to stall when the photospheric driving is supressed during non-eruptive stages. Still, the late onset of this reconnection clearly accelerates eruptions in some 2.5D and 3D MHD simulations. But these eruptions were previously initiated by another mechanism, such as the magnetic breakout process (Lynch et al. 2008; Karpen et al. 2012, as described hereafter) and ideal instabilities 
(Nishida et al. 2013). Some 3D flux emergence simulations did report a qualitative role for tether-cutting reconnection in their eruptions (Manchester et al. 2004; Archontis \& Hood 2008). But they did not show that it was explicitly driving the eruptions.

So the tether-cutting has never been proven to initiate, alone, an eruption in any $3 \mathrm{D}$ simulations. This negative result was found (but rarely written) by independent groups using different codes. This raises strong doubts about the validity of the tether-cutting as an eruption driving mechanism. However, this reconnection is obviously an important aspect of every solar eruption. Indeed it provides an extra-acceleration to the erupting prominence and, of course, it releases a lot of magnetic energy and it produces the most energetic particles in the flare that develops in the wake of the CME (Masson et al. 2013).

\subsection{Magnetic breakout}

A new idea was proposed by Antiochos et al. (1999), for lowering the flux and the tension of the overlying arcades, by invoking magnetic reconnection occurring at a magnetic null point, being located at high altitude above the current-carrying field lines.

Observationally, this model requires a quadrupolar topology for the photospheric magnetic field. This condition can be satisfied in many active regions, especially young ones (see e.g. Ugarte-Urra et al. 2007). But is not guaranteed for older decaying active regions that look bipolar (see e.g. van Driel-Gesztelyi et al. 2003), although large remote connections may still be invoked. Theoretically, Antiochos et al. (1999) proved for axisymmetric systems that this "magnetic breakout" alone can drive eruptions, provided that the onset of null point reconnection was delayed during the slow energy build-up phase, and that the rate of reconnection was slow enough during the fast eruptive phase. As for the tethercutting reconnection, the breakout reconnection could create a feedback-loop, leading to an explosive behavior, hence to an eruption. In addition, DeVore \& Antiochos (2005) found that the efficiency of this mechanism also depends on the ratio of the magnetic fluxes located above and below the null point: if the flux of the largest overlying arcades is too weak (resp. too strong), there is not enough (resp. too much) flux to reconnect for the breakout mechanism to be sustained long enough for a full eruption.

Full simulations of the breakout were first achieved in 2.5D MHD simulations (MacNeice et al. 2004), including with very high spatial resolutions (Karpen et al. 2012; Lynch \& Edmondson 2013) and with the solar wind (van der Holst et al. 2007; Masson et al. 2013). A key difference with the tether-cutting model, though, is that the breakout was also found to occur in a 3D line-tied simulation (Lynch et al. 2008), and very probably in a 3D flux emergence simulation (Archontis \& Török 2008). Also, this original MHD model found unambiguous support in several observational analyses (e.g. Aulanier et al. 2000; Sterling \& Moore 2001; Gary \& Moore 2004; Ugarte-Urra et al. 2007).

So the breakout mechanism appears as a robust process to initiate and drive solar eruptions, although it has several requirements that prevents it from being general (DeVore $\&$ Antiochos 2005), and it may require a relatively strong flare reconnection to produce a fast eruption (Karpen et al. 2012; Masson et al. 2013).

\subsection{Side-reconnections and remote couplings}

Some other models also explain eruptions through coronal reconnection, which occurs aside of the prominence instead of below or above it. In several cases this reconnection can increase the length of the overlying arcades, and lower their tension.

Eruptions driven by this process were modeled in the context of small-scale flux emergence in the vicinity of the flux rope, in the $2 \mathrm{D}$ electric-wire paradigm (Lin et al. 2001), in 2.5D MHD simulations (Chen \& Shibata 2000) and recently in 3D simulations (Kusano et al. 2012; Toriumi et al. 2013). It was also found to operate in 3D MHD models 
of interacting active regions (Jacobs et al. 2009), and possibly to trigger sympathetic eruptions in models where several current-carrying flux tubes are included (Török et al. 2011; Lynch \& Edmondson 2013), in line with the concept proposed by Schrijver \& Title (2011) and further developed by Schrijver et al. (2013).

The dominant mechanism that drive eruptions in these side-reconnection models is still uncertain. Chen \& Shibata (2000) attributes the eruption to the tether-cutting reconnection triggered by the side-reconnection. The broad coverage of the paramater space achieved by Kusano et al. (2012) shows that the eruptivity strongly depends on the magnetic field configuration. Schrijver et al. (2013) and Lynch \& Edmondson (2013) argue that eruptions are triggered sympathetically because the corona is constantly reconfiguring from the previous eruption. Lin et al. (2001) show that an ideal loss of equilibrium is triggered in the new system that results from the appearance of a new bipole. And finally Török et al. (2011) show that the coronal reconfiguration that results from high-altitude reconnection, actually leads pre-eruptive flux ropes that are almost torus-unstable to enter the instability regime and then erupt one after the other.

So, like in flux emergence models, a loss of equilibrium / torus instability can be triggered by remote reconnections that result in small-scale or large-scale couplings. But other interpretations for the cause of the eruptions have also been proposed.

\subsection{Converging motions}

Models driven by photospheric motions that converge toward polarity inversion lines, above which prominences are located, have also been considered. Quasi-static theory shows that reducing the length-scale of the photospheric magnetic field also reduces the magnitude of the coronal field at large heights, and makes the field drop faster with height. A priori, both can facilitate the torus instability.

This is strongly suggested by the landmark electric-wire model by Forbes \& Priest (1995) and by the MHD simulations of Török \& Kliem (2007). They explored eruptive behaviors, by making several independent calculations for different ratios between the current or the height of the pre-eruptive flux rope, and the horizontal extent of the surrounding photospheric bipolar field.

Eruptions of current-carrying fields subject to dynamically-treated converging motions, have also been found in MHD simulations, both in 2.5D (Inhester et al. 1992) and in 3D (Amari et al. 2003a). Recently, a direct MHD simulation of an observed event, forced by ideal converging motions, also produced an eruption (Zuccarello et al. 2012).

Such motions are frequently observed at the Sun's surface. This makes the model appealing. Some questions remain open, though: very extended motions as used in the models are rarely observed; and the physical mechanism that actually drives the eruptions has not yet been firmly identified in the MHD models.

\subsection{Decreasing photospheric magnetic field}

This class of models can somehow be viewed as the exact opposite as the axial flux increase models. They rely on a homogeneous magnetic field decrease in an extended section of the photosphere around the flux rope. This decrease is imposed, either by reducing the magnetic momentum of the external subphotospheric magnetic field sources (see e.g. Lin et al. 1998), or by prescribing adequate horizontal electric fields in the photosphere (see e.g. Amari et al. 2000). In axisymmetric geometry, this process produces eruptions of detached flux ropes, that are either pre-existing (Lin et al. 1998), or slowly formed during the magnetic field decrease (Linker et al. 2003; Reeves et al. 2010). It can also form and trigger the eruption of line-tied flux ropes of various sizes in 3D (Amari et al. 2000; Lin et al. 2002; Linker et al. 2003). 
Qualitatively, the origin of the eruptions can be directly attributed to the diminishing of the coronal restraining tension, that naturally results from the gradual disappearance of the photospheric magnetic flux. Quantitatively, the eruptions occur when the diminishing magnetic energy of the fully open field reaches down to a value that is equal to that of the current-carrying fields, as identified by Amari et al. (2000). This interpretation is particularly interesting because it provides a very clear eruption threshold in the correct MHD paradigm (like the torus instability does in the electric-wire paradigm).

The physical validity of these models is still debated, as it is difficult to find a selfconsistent MHD process that diminishes the photospheric magnetic field over large areas. Amari et al. (2000) qualitatively noted that flux rope emergence can actually lead to an apparent flux decrease on the side rope, after the emergence of the rope axis. But it is unclear whether this process produces the magnetic field decrease as required for an eruption. Amari et al. (2010) quantitatively calculated that photospheric flows that mimic flux dispersal in decaying active regions (as described below) can account for the prescribed flux decrease. But this interpretation requires flows that accelerate to infinite speeds towards the polarity inversion line, which may be problematic.

\subsection{Flux dispersal and cancellation}

The "flux-cancellation" model is based on the observed long-term evolution of magnetic flux concentrations in the photosphere, within or between bipolar active regions (e.g. Wang et al. 1989; Démoulin et al. 2002; van Driel-Gesztelyi et al. 2003; Schmieder et al. 2008; Green et al. 2011). Over time-periods of days to months, depending on their sizes, flux concentrations disperse and spread in all directions. Their apparent diffusion leads their peak and mean magnetic field magnitude to decrease, while their total magnetic flux slowly and weakly decreases through local flux convergence and cancellation at polarity inversion lines, right below prominences.

The landmark references for this model are van Ballegooijen \& Martens (1989) and Forbes \& Isenberg (1991). They showed that converging motions and flux cancellation combined all together (with no flux dispersal or decrease) yield the gradual formation of a flux rope through a tether-cutting-like photospheric reconnection, that involves coronal arcades that have previously been sheared in a $2.5 \mathrm{D}$ geometry. On the long run, the rope grows in size and in altitude until it erupts, as calculated in $2.5 \mathrm{D}$ electric-wire models (Forbes \& Isenberg 1991; Isenberg et al. 1993).

By treating the large-scale decay of the photospheric magnetic field with an extra photospheric diffusion term in the induction equation (as introduced by Wang et al. 1989), 3D flux ropes were also found to form and erupt, firstly by Amari et al. (2003b) with MHD simulations, and later by Mackay \& van Ballegooijen (2006) and Yeates \& Mackay (2009) with magneto-frictional simulations. These results were also found in non-symmetric MHD models (Aulanier et al. 2010, 2012; Pagano et al. 2013), and in symmetric models in which the flux dispersal was instead treated by line-tied flows diverging from the center each flux concentration (Amari et al. 2011).

The magnetic flux decrease model (see Sect. 4.5) is often regarded as a flux cancellation model. But both are physically very different. Firstly, in the flux cancellation model the magnetic flux decreases locally because of magnetic field annihilation at the inversion line (Wang et al. 1989). That is different than a flux decrease induced by a diminishing magnetic field over a large area. Secondly, the flux cancellation model does not involve a dimishing of the open field energy down to the magnetic field energy of the pre-eruptive field (see Amari et al. 2003b).

Detailed analysis of one MHD simulation, and its comparison with electric-wire models, showed that "photospheric flux-cancellation and tether-cutting coronal reconnection do 
not trigger CMEs in bipolar magnetic fields, but are key pre-eruptive mechanisms for flux ropes to build up and to rise to the critical height above the photosphere at which the torus instability causes the eruption" (Aulanier et al. 2010).

\section{Discussion}

A large variety of storage-and-release eruption models have been developed during the last forty years. At first sight they look similar to each other. Indeed, they predict similar observable features, and they share common physical ingredients. But they also contain important differences, either in their equations, their geometries, and their prescriptions. So they have often been opposed to each other. This emulation fostered fine-tuned developments and analyses, up to a stage at which they can now be classified and compared with one another, so that the physical mechanisms that initiate, drive, and contribute to prominence eruptions may now be identified independently of the models themselves.

When acknowledging that prominence eruptions occur once the magnetic pressure explosively wins over the magnetic tension (exerted on the system by the overlying coronal arcades), then the present review along with that of Schmieder et al. (2013) suggest that, to date, no more than two physical mechanisms can initiate and sustain this explosive loss of force balance.

The ideal loss of equilibrium of a flux rope is the first mechanism. 3D models have shown that the prominence flux rope does not actually need to be very twisted: the mechanism works with ropes that have less than one turn. The eruption there occurs once the rope axis has reached an altitude, above which all stable equilibria cease to exist. The threshold is reached when the magnitude of magnetic fields of the overlying arcades decrease faster with height than the magnetic pressure which pushes the flux rope upwards, which also decreases with time during the rise of the flux rope. The process was first proposed by van Tend \& Kuperus (1978), and it was shown by Démoulin \& Aulanier (2010) to correspond to the "torus instability" first proposed by Bateman (1978) in tokamaks, and first revisited for solar eruptions by Kliem \& Török (2006).

The removal of the arcades that overlay and confine the prominence by means of highaltitude magnetic reconnection is the second mechanism. It has initially been proposed to occur at null points, but it may also operate at separators and quasi-separatrix layers. Once the reconnection has begun, it transfers overlying arcades into connectivity domains that are located aside of the prominence. So the amount of magnetic flux that overlays the prominence is reduced. The associated diminishing of the confinement makes the prominence rise to larger altitudes. This provides a loop-feedback on the high-altitude reconnection, so that an eruption can occur. This process, called the "magnetic breakout", was first proposed by Antiochos et al. (1999). The efficiency of the breakout requires the magnetic fluxes located above and below the reconnection region be comparable in magnitude (DeVore \& Antiochos 2005).

To date, only the torus instability and the magnetic breakout were found to occur in many different 3D MHD simulations. The torus instability has been identified to cause eruptions with prescribed unstable flux ropes (Roussev et al. 2003; Török \& Kliem 2005, 2007; Schrijver et al. 2008; Török et al. 2010), with kinematic flux emergence (Fan \& Gibson 2007; Fan 2010), with flux cancellation (Aulanier et al. 2010, 2012), with sympathetically erupting flux ropes (Török et al. 2011), and with non-linear force-free relaxations (Kliem et al. 2013; Jiang et al. 2013). The magnetic breakout mechanism has been shown to operate with shearing bipoles in multipolar geometry (Lynch et al. 2008), with twisted flux tubes emerging through a stratified medium into a pre-existing horizontal field (Archontis \& Török 2008; MacTaggart \& Hood 2009b), and with sympathetically erupting 
sheared loops (Lynch \& Edmondson 2013). So it can be conjectured that eruptions can only be initiated and driven by one of these two mechanisms, or their combination.

It follows that, depending on the solar conditions, all the other processes may be considered as different ways to either bring the system to the threshold of one of these two mechanisms, or to help making the resulting eruption faster. For example, flux emergence (Fan 2010) or flux cancellation (Aulanier et al. 2010) can initiate a torus instability. Also, reconnection-driven long-range couplings around flux ropes (Török et al. 2011) or sheared arcades (Lynch \& Edmondson 2013) can initiate sympathetic torus instabilities and magnetic breakouts. And flare reconnection can accelerate eruptions initiated by a torus instability (Nishida et al. 2013) and a magnetic breakout (Karpen et al. 2012; Masson et al. 2013).

In this line each and every solar process that can contribute to solar eruptions should be taken into account, all together with the few physical mechanisms that initiate and drive eruptions, so as to reach a comprehensive understanding of observed events, and so as to predict the occurrence of future events.

\section{References}

Aly, J. J. 1984, Astrophys. J., 283, 349

-. 1985, Astron. Astrophys., 143, 19

-. 1991, Astrophys. J. Lett., 375, L61

Amari, T. \& Aly, J. J. 1990, Astron. Astrophys., 227, 628

Amari, T., Aly, J.-J., Luciani, J.-F., Mikic, Z., \& Linker, J. 2011, Astrophys. J. Lett., 742, L27

Amari, T., Aly, J.-J., Mikic, Z., \& Linker, J. 2010, Astrophys. J. Lett., 717, L26

Amari, T. \& Luciani, J. F. 1999, Astrophys. J. Lett., 515, L81

Amari, T., Luciani, J. F., \& Aly, J. J. 2004, Astrophys. J. Lett., 615, L165

-. 2005, Astrophys. J. Lett., 629, L37

Amari, T., Luciani, J. F., Aly, J. J., Mikic, Z., \& Linker, J. 2003a, Astrophys. J., 585, 1073

-. 2003b, Astrophys. J., 595, 1231

Amari, T., Luciani, J. F., Aly, J. J., \& Tagger, M. 1996a, Astron. Astrophys., 306, 913

-. 1996b, Astrophys. J. Lett., 466, L39

Amari, T., Luciani, J. F., Mikic, Z., \& Linker, J. 2000, Astrophys. J. Lett., 529, L49

Antiochos, S. K., Dahlburg, R. B., \& Klimchuk, J. A. 1994, Astrophys. J. Lett., 420, L41

Antiochos, S. K., DeVore, C. R., \& Klimchuk, J. A. 1999, Astrophys. J., 510, 485

Archontis, V. \& Hood, A. W. 2008, Astrophys. J. Lett., 674, L113

-. 2012, Astron. Astrophys., 537, A62

Archontis, V., Hood, A. W., Savcheva, A., Golub, L., \& Deluca, E. 2009, Astrophys. J., 691, 1276

Archontis, V., Moreno-Insertis, F., Galsgaard, K., Hood, A., \& O'Shea, E. 2004, Astron. Astrophys., 426, 1047

Archontis, V. \& Török, T. 2008, Astron. Astrophys., 492, L35

Aulanier, G., DeLuca, E. E., Antiochos, S. K., McMullen, R. A., \& Golub, L. 2000, Astrophys. J., 540, 1126

Aulanier, G., Démoulin, P., \& Grappin, R. 2005, Astron. Astrophys., 430, 1067

Aulanier, G., DeVore, C. R., \& Antiochos, S. K. 2002, Astrophys. J. Lett., 567, L97

Aulanier, G., Janvier, M., \& Schmieder, B. 2012, Astron. Astrophys., 543, A110

Aulanier, G., Török, T., Démoulin, P., \& DeLuca, E. E. 2010, Astrophys. J., 708, 314

Bateman, G. 1978, MHD instabilities, ed. G. Bateman

Birn, J., Goldstein, H., \& Schindler, K. 1978, Solar Phys., 57, 81

Chen, J. 1989, Astrophys. J., 338, 453

Chen, P. F. \& Shibata, K. 2000, Astrophys. J., 545, 524

Démoulin, P. \& Aulanier, G. 2010, Astrophys. J., 718, 1388

Démoulin, P., Mandrini, C. H., van Driel-Gesztelyi, L., Thompson, B. J., Plunkett, S., Kovári, Z., Aulanier, G., \& Young, A. 2002, Astron. Astrophys., 382, 650 
DeVore, C. R. \& Antiochos, S. K. 2000, Astrophys. J., 539, 954

-. 2005, Astrophys. J., 628, 1031

Emonet, T. \& Moreno-Insertis, F. 1998, Astrophys. J., 492, 804

Fan, Y. 2001, Astrophys. J. Lett., 554, L111

-. 2010, Astrophys. J., 719, 728

Fan, Y. \& Gibson, S. E. 2004, Astrophys. J., 609, 1123

-. 2007, Astrophys. J., 668, 1232

Filippov, B. P. \& Den, O. G. 2001, Journal of Geophysical Research, 106, 25177

Forbes, T. G. 1990, Journal of Geophysical Research, 95, 11919

-. 2000, Journal of Geophysical Research, 105, 23153

Forbes, T. G. \& Isenberg, P. A. 1991, Astrophys. J., 373, 294

Forbes, T. G., Linker, J. A., Chen, J., Cid, C., Kóta, J., Lee, M. A., Mann, G., Mikić, Z., Potgieter, M. S., Schmidt, J. M., Siscoe, G. L., Vainio, R., Antiochos, S. K., \& Riley, P. 2006, Space Science Reviews, 123, 251

Forbes, T. G. \& Priest, E. R. 1995, Astrophys. J., 446, 377

Gary, G. A. \& Moore, R. L. 2004, Astrophys. J., 611, 545

Georgoulis, M. K., Titov, V. S., \& Mikić, Z. 2012, Astrophys. J., 761, 61

Green, L. M., Kliem, B., \& Wallace, A. J. 2011, Astron. Astrophys., 526, A2

Heyvaerts, J., Lasry, J. M., Schatzmann, M., \& Witomsky, P. 1982, Astron. Astrophys., 111, 104

Inhester, B., Birn, J., \& Hesse, M. 1992, Solar Phys., 138, 257

Inoue, S. \& Kusano, K. 2006, Astrophys. J., 645, 742

Isenberg, P. A. \& Forbes, T. G. 2007, Astrophys. J., 670, 1453

Isenberg, P. A., Forbes, T. G., \& Démoulin, P. 1993, Astrophys. J., 417, 368

Isobe, H., Tripathi, D., \& Archontis, V. 2007, Astrophys. J. Lett., 657, L53

Jacobs, C., Poedts, S., \& van der Holst, B. 2006, Astron. Astrophys., 450, 793

Jacobs, C., Roussev, I. I., Lugaz, N., \& Poedts, S. 2009, Astrophys. J. Lett., 695, L171

Jiang, C., Feng, X., Wu, S. T., \& Hu, Q. 2013, Astrophys. J. Lett., 771, L30

Jouve, L. \& Brun, A. S. 2009, Astrophys. J., 701, 1300

Karpen, J. T., Antiochos, S. K., \& DeVore, C. R. 2012, Astrophys. J., 760, 81

Kliem, B., Su, Y., van Ballegooijen, A., \& DeLuca, E. 2013, ArXiv e-prints

Kliem, B. \& Török, T. 2006, Physical Review Letters, 96, 255002

Klimchuk, J. A. \& Sturrock, P. A. 1989, Astrophys. J., 345, 1034

Kusano, K., Bamba, Y., Yamamoto, T. T., Iida, Y., Toriumi, S., \& Asai, A. 2012, Astrophys. J., 760,31

Leake, J. E., Linton, M. G., \& Antiochos, S. K. 2010, Astrophys. J., 722, 550

Lin, J. \& Forbes, T. G. 2000, Journal of Geophysical Research, 105, 2375

Lin, J., Forbes, T. G., \& Isenberg, P. A. 2001, Journal of Geophysical Research, 106, 25053

Lin, J., Forbes, T. G., Isenberg, P. A., \& Démoulin, P. 1998, Astrophys. J., 504, 1006

Lin, J., van Ballegooijen, A. A., \& Forbes, T. G. 2002, Journal of Geophysical Research (Space Physics), 107, 1438

Linker, J. A., Mikić, Z., Lionello, R., Riley, P., Amari, T., \& Odstrcil, D. 2003, Physics of Plasmas, 10, 1971

Low, B. C. 1977, Astrophys. J., 212, 234

Lynch, B. J., Antiochos, S. K., DeVore, C. R., Luhmann, J. G., \& Zurbuchen, T. H. 2008, Astrophys. J., 683, 1192

Lynch, B. J. \& Edmondson, J. K. 2013, Astrophys. J., 764, 87

Mackay, D. H. \& van Ballegooijen, A. A. 2006, Astrophys. J., 641, 577

MacNeice, P., Antiochos, S. K., Phillips, A., Spicer, D. S., DeVore, C. R., \& Olson, K. 2004, Astrophys. J., 614, 1028

MacTaggart, D. \& Hood, A. W. 2009a, Astron. Astrophys., 501, 761

-. 2009b, Astron. Astrophys., 508, 445

-. 2009c, Astron. Astrophys., 507, 995

Magara, T. \& Longcope, D. W. 2001, Astrophys. J. Lett., 559, L55

Manchester, IV, W., Gombosi, T., DeZeeuw, D., \& Fan, Y. 2004, Astrophys. J., 610, 588 
Martens, P. C. H. \& Kuin, N. P. M. 1989, Solar Phys., 122, 263

Masson, S., Antiochos, S. K., \& DeVore, C. R. 2013, Astrophys. J., 771, 82

Mikic, Z. \& Linker, J. A. 1994, Astrophys. J., 430, 898

Molodenskii, M. M. \& Filippov, B. P. 1987, Soviet Astronomy, 31, 564

Moore, R. L. \& Roumeliotis, G. 1992, Lecture Notes in Physics, Springer Verlag, IAU Colloq. 133: Eruptive Solar Flares, 399, 69

Moore, R. L., Sterling, A. C., Hudson, H. S., \& Lemen, J. R. 2001, Astrophys. J., 552, 833

Nishida, K., Nishizuka, N., \& Shibata, K. 2013, Astrophys. J. Lett., 775, L39

Olmedo, O. \& Zhang, J. 2010, Astrophys. J., 718, 433

Olmedo, O., Zhang, J., \& Kunkel, V. 2013a, Astrophys. J., 771, 125

Olmedo, O., Zhang, J., \& Chen, J. 2013b, Astrophys. J., submitted

Pagano, P., Mackay, D. H., \& Poedts, S. 2013, Astron. Astrophys., 554, A77

Pariat, E., Aulanier, G., Schmieder, B., Georgoulis, M. K., Rust, D. M., \& Bernasconi, P. N. 2004, Astrophys. J., 614, 1099

Rachmeler, L. A., DeForest, C. E., \& Kankelborg, C. C. 2009, Astrophys. J., 693, 1431

Reeves, K. K., Linker, J. A., Mikić, Z., \& Forbes, T. G. 2010, Astrophys. J., 721, 1547

Roumeliotis, G., Sturrock, P. A., \& Antiochos, S. K. 1994, Astrophys. J., 423, 847

Roussev, I. I., Forbes, T. G., Gombosi, T. I., Sokolov, I. V., DeZeeuw, D. L., \& Birn, J. 2003, Astrophys. J. Lett., 588, L45

Schmieder, B., Bommier, V., Kitai, R., Matsumoto, T., Ishii, T. T., Hagino, M., Li, H., \& Golub, L. 2008, Solar Phys., 247, 321

Schmieder, B. \& Aulanier, G. 2012, Advances in Space Research, 49, 1598

Schmieder, B., Démoulin, P., \& Aulanier, G. 2013, Advances in Space Research, 51, 1967

Schrijver, C. J., Elmore, C., Kliem, B., Török, T., \& Title, A. M. 2008, Astrophys. J., 674, 586

Schrijver, C. J. \& Title, A. M. 2011, Journal of Geophysical Research (Space Physics), 116, 4108

Schrijver, C. J., Title, A. M., Yeates, A. R., \& DeRosa, M. L. 2013, Astrophys. J., 773, 93

Shafranov, V. D. 1966, Reviews of Plasma Physics, 2, 103

Sterling, A. C. \& Moore, R. L. 2001, Astrophys. J., 560, 1045

Sturrock, P. A. 1989, Solar Phys., 121, 387

-. 1991, Astrophys. J., 380, 655

Sturrock, P. A., Antiochos, S. K., \& Roumeliotis, G. 1995, Astrophys. J., 443, 804

$\mathrm{Su}$, Y., Surges, V., van Ballegooijen, A., DeLuca, E., \& Golub, L. 2011, Astrophys. J., 734, 53

Titov, V. S. \& Démoulin, P. 1999, Astron. Astrophys., 351, 707

Toriumi, S., Iida, Y., Bamba, Y., Kusano, K., Imada, S., \& Inoue, S. 2013, Astrophys. J., 773, 128

Török, T., Berger, M. A., \& Kliem, B. 2010, Astron. Astrophys., 516, A49

Török, T. \& Kliem, B. 2003, Astron. Astrophys., 406, 1043

-. 2005, Astrophys. J. Lett., 630, L97

-. 2007, Astronomische Nachrichten, 328, 743

Török, T., Panasenco, O., Titov, V. S., Mikić, Z., Reeves, K. K., Velli, M., Linker, J. A., \& De Toma, G. 2011, Astrophys. J. Lett., 739, L63

Ugarte-Urra, I., Warren, H. P., \& Winebarger, A. R. 2007, Astrophys. J., 662, 1293

van Ballegooijen, A. A. 2004, Astrophys. J., 612, 519

van Ballegooijen, A. A. \& Martens, P. C. H. 1989, Astrophys. J., 343, 971

van der Holst, B., Jacobs, C., \& Poedts, S. 2007, Astrophys. J. Lett., 671, L77

van Driel-Gesztelyi, L., Démoulin, P., Mandrini, C. H., Harra, L., \& Klimchuk, J. A. 2003, Astrophys. J., 586, 579

van Tend, W. 1979, Solar Phys., 61, 89

van Tend, W. \& Kuperus, M. 1978, Solar Phys., 59, 115

Wang, Y.-M., Nash, A. G., \& Sheeley, Jr., N. R. 1989, Science, 245, 712

Yeates, A. R. \& Mackay, D. H. 2009, Astrophys. J., 699, 1024

Zuccarello, F. P., Meliani, Z., \& Poedts, S. 2012, Astrophys. J., 758, 117

Zwingmann, W. 1987, Solar Phys., 111, 309 